\title{
Erradicación de Helicobacter pylori con terapia triple estándar de primera línea
}

\author{
Helicobacter pylori eradication with standard first-line triple therapy \\ Diego R. Menéndez-Morales ${ }^{1}$, Roberto R. Chapas-González, \\ María R. Ligorría-Goicolea², Luis C. Barrios-Lupitou ${ }^{3 *}$ \\ ${ }^{1}$ Departamento de Medicina Interna y ${ }^{2}$ Unidad de Gastroenterología, Hospital General San Juan de Dios, \\ Guatemala, Guatemala, y ${ }^{3}$ Facultad de Ciencias Médicas, Universidad de San Carlos de Guatemala, Guatemala.
}

*Autor al que se dirige la correspondencia: carlos.barrios@hotmail.com

Recibido: 11 de mayo 2017 / Revisión: 20 de marzo 2018 / Aceptado: 31 de mayo 2018

Resumen

$\mathrm{L}$ a infección por Helicobacter pylori es una de las infecciones crónicas más comunes a nivel mundial y causa importante de enfermedad péptica y cáncer gástrico. Infecta al $50 \%$ de la población adulta con mayor prevalencia en América Central/Sur y Asia y al menos dos veces mayor en poblaciones con alta incidencia de cáncer gástrico. Los objetivos de esta investigación fueron identificar la tasa de erradicación de H. pylori con terapia triple estándar y las posibles características asociadas a su erradicación. Se estudió a 119 pacientes con diagnóstico de infección por $H$. pylori, seleccionados en forma consecutiva de la consulta externa de Gastroenterología del Hospital General San Juan de Dios. Se realizó endoscopia diagnóstica y toma de biopsia gástrica. Se dio terapia triple estándar con lansoprazol, amoxicilina y claritromicina durante 10 días, seguido de 30 días con lansoprazol. Seis semanas después de completado el tratamiento se evaluó el antígeno de $H$. pylori en heces para determinar si hubo erradicación. La edad promedio de los participantes fue 49.0 años, 81.5\% mujeres, 85.7\% de área urbana, el síntoma más común fue dispepsia en 86.6\%. En el examen post tratamiento el 89.9\%, IC 95\% [83.0, 94.7] presentó antígeno en heces negativo. No se encontró asociación entre las características de los pacientes con la respuesta al tratamiento. En conclusión, la respuesta a la terapia triple de primera línea se encuentra dentro del rango aceptable para continuar con ese esquema, pero debe mantenerse una evaluación constante por la presencia de posible resistencia.

Palabras claves: Dispepsia, pirosis, gastritis, neoplasias gástricas

\section{Abstract}

$\mathrm{I}^{\mathrm{n}}$ nfection by Helicobacter pylori is one of the most common chronic infections worldwide and an important cause of peptic 1 disease and gastric cancer. It infects $50 \%$ of the adult population with the highest prevalence in Central / South America and Asia and at least twice as high in populations with a high incidence of gastric cancer. The objectives of this research were to identify the eradication rate of $H$. pylori after first line standard triple therapy and the possible characteristics associated with its eradication. It was studied 119 patients with a diagnosis of $H$. pylori infection, selected consecutively from the outpatient department of Gastroenterology of the San Juan de Dios General Hospital. Diagnostic endoscopy and gastric biopsy was performed. Standard triple therapy was given with lansoprazole, amoxicillin and clarithromycin for 10 days, followed by 30 days with lansoprazole. Six weeks after the treatment was completed, the $H$. pylori antigen in feces was evaluated to determine if there was eradication. The average age of the participants was 49.0 years, $81.5 \%$ women, $85.7 \%$ of urban area, the most common symptom was dyspepsia in $86.6 \%$. In the post-treatment examination $89.9 \%, 95 \%$ CI [83.0, 94.7] presented negative antigen in feces. No association was found between the characteristics of the patients with the response to treatment. In conclusion, the response to first line triple therapy is within the acceptable range to continue with this scheme, but a constant evaluation must be maintained due to the presence of possible resistance.

Keywords Dyspepsia, heartburn, gastritis, stomach neoplasms 


\section{Introducción}

La infección por Helicobacter pylori se considera una de las infecciones bacterianas crónicas más prevalentes en humanos, en países desarrollados la prevalencia es del 30 al $50 \%$ y en los subdesarrollados del 50 al $80 \%$, con una tendencia a disminuir en los desarrollados (Benajah et al., 2013; Mentis, Lehours, \& Mégraud, 2015). Los factores de riesgo más importantes son las condiciones socioeconómicas y la niñez (Eusebi, Zagari, \& Bazzoli, 2014). La prevalencia general es alta en países en vías de desarrollo, con una marcada diferencia entre las poblaciones urbanas y rurales (Schneider et al., 1994). En Guatemala en adultos es del 65 a $80 \%$ y constituye un problema de salud pública, por lo que su erradicación es de vital importancia (Hunt et al., 2011).

El Colegio Americano de Gastroenterología (Chey, Wong, \& the Practice Parameters Committee of the American College of Gastroenterology, 2007), el Segundo Consenso Asia-Pacífico (Fock et al., 2009) y el Consenso Maastrich IV y Florencia (Malfertheiner et al., 2012) para el tratamiento de la infección por $H$. pylori, recomiendan tratar a todo paciente con prueba positiva debido a mayor asociación en el desarrollo de úlceras gástricas o duodenales (reportada entre 1 y $10 \%$ de los pacientes infectados), cáncer gástrico (del 0.1 al $3 \%$ ) y linfoma gástrico MALT ( $m$ ucosa associated lymphoid tissue, en inglés) (en $0.01 \%$ ), por lo que el tratamiento oportuno con un adecuado esquema terapéutico podría tener un importante efecto epidemiológico.

Se estima que la infección por $H$. pylori es causa del 5.2 al 6.2\% de todos los cánceres (Parkin, 2006; Plummer, Franceschi, Vignat, Forman, \& De Martel, 2015).

La erradicación depende de diversos factores (O'Connor, Vaira, Gisbert, \& O'Morain, 2014) entre los que incluye los genéticos (Suzuki et al., 2006) y la adherencia al tratamiento (Fischbach, Van Zanten, \& Dickason, 2004). La resistencia al tratamiento se reporta muy variable según la región o país, con $50 \%$ en China, $40 \%$ en Turquía, 30\% en Japón e Italia, y bajas como Suecia y Taiwán con $15 \%$ (Thung et al., 2016).

La evaluación de la erradicación de la infección por $H$. pylori se puede realizar por diferentes métodos como la prueba de urea en aliento, de la biopsia por endoscopía, cultivo de biopsia gástrica y el antígeno en heces (Chey, Leontiadis, Howden, \& Moss, 2017; Garza-González, Perez-Perez, Maldonado-Garza, \& Bosques-Padilla, 2014; Garza González et al., 2007;
Li et al., 2015; Prell et al., 2009), cada una con sus ventajas y desventajas.

En Guatemala se ha reportado tasas de resistencia altas para algunos antibióticos como metronidazol y claritromicina, hasta 42\% (Barahona-Garrido, Ortiz, \& Sarti, 2012), por lo que recomiendan el uso de otros esquemas (Barahona-Garrido, Quiñonez, Cerda-Contreras, Maria Sarti, \& Téllez-Ávila, 2013).

El conocimiento sobre la respuesta al tratamiento de erradicación de esta bacteria puede ayudar al tratamiento dirigido a cierto grupo poblacional y de esta manera se podrá identificar el esquema adecuado a nuestro contexto. Los objetivos de esta investigación fueron identificar la tasa de erradicación de $H$. pylori en pacientes que reciben terapia triple estándar de acuerdo a antígeno presente en heces (Chey et al., 2007; Garza González et al., 2007) e identificar posibles características asociadas (sexo, grupo etario y síntomas más comunes) a la erradicación.

\section{Materiales y métodos}

Estudio descriptivo longitudinal. Se estudió a 125 pacientes con diagnóstico de $\mathrm{H}$. pylori, seleccionados en forma consecutiva en la consulta externa de Gastroenterología del Hospital General San Juan de Dios. Se incluyó a pacientes de ambos sexos, de 18 años de edad o más, que tuvieran síntomas o signos sugestivos de infección por H. pylori (dispepsia, pérdida de peso inexplicable, distensión abdominal, pirosis, dolor en epigastrio, vómitos persistentes, saciedad temprana, hemorragia manifestada como heces con sangre o vómitos con sangre), con biopsia positiva para H. pylori.

Se consideró como criterios de exclusión si habían recibido tratamiento previo para erradicación de H. pylori; enfermedades que confieren algún grado de inmunosupresión (diabetes mellitus, enfermedades autoinmunes, infección por VIH, enfermedades hematológicas, neoplasias, uso crónico de esteroides, exposición a quimioterapia o radioterapia); embarazo; uso de antibióticos en las últimas cuatro semanas; alergia a alguno de los componentes utilizados en los esquemas de erradicación a emplearse en el estudio (penicilinas, claritromicina, quinolonas); rechazo a participar en el estudio; que no finalizaran el tratamiento a las dosis adecuadas durante los 10 días; que no se realizaran el examen en heces por cualquier motivo e información incompleta. 
Se seleccionó a 219 pacientes, 211 asistieron a realizarse la endoscopia, 125 presentaron biopsia positiva para la infección por H. pylori, de los cuales 119 recibieron tratamiento. Se excluyó a seis pacientes por no cumplir los criterios previamente establecidos (tratamiento incompleto por presentar efectos adversos al tratamiento, cáncer gástrico avanzado, diabetes mellitus, embarazo y rechazo a tomar el tratamiento) (Figura 1).

Para la recolección de los datos se entrevistó a todos los pacientes que acudieron a la consulta externa de Gastroenterología del Hospital General San Juan de Dios de febrero a julio de 2016 y que tenían alguna indicación para realizar endoscopía por signos o síntomas sugestivos de infección por H. pylori. Se registró datos generales como nombre, edad, dirección y si contaban con servicios básicos (drenajes, agua, luz) para categorizar la procedencia como urbana o rural.

Basado en las Guías para el tratamiento de la infección por H. pylori del Segundo Consenso de Asia-Pacífico (Fock et al., 2009) y el Consenso Global de Kyoto (Sugano et al., 2015), se realizó endoscopia y toma de biopsia gástrica, se entregó a cada paciente el informe de la endoscopía y el resultado de la biopsia. A los pacientes con resultado positivo para $H$. pylori en biopsia se inició tratamiento para erradicación con terapia triple (lansoprazol $30 \mathrm{mg}$ por vía oral dos veces al día, amoxicilina $1 \mathrm{~g}$ por vía oral dos veces al día y claritromicina $500 \mathrm{mg}$

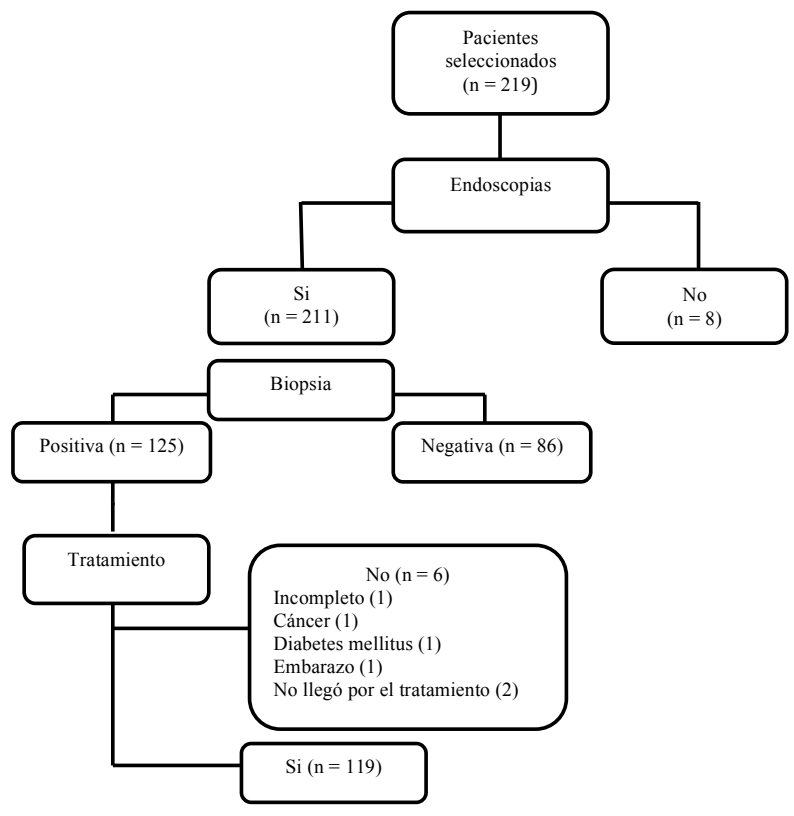

por vía oral dos veces al día) durante 10 días, seguido de 30 días de tratamiento con lansoprazol.

Se procuró asegurar la adherencia al tratamiento, para ello se localizó vía telefónica a los pacientes para brindarles el resultado de la biopsia y se prescribía el medicamento; una vez recetado el tratamiento, se monitoreaba vía telefónica y si el paciente presentaba dudas o alguna sintomatología se citaba para evaluar si podía continuar con el tratamiento.

Se citó a los pacientes seis semanas después de completado el tratamiento para realizar antígeno de $H$. pylori en heces, para determinar si hubo erradicación (Chey et al., 2007; Garza González et al., 2007; Malfertheiner et al., 2017). Las muestras fueron analizadas por inmunoensayo cromatográfico $\mathrm{Abon}^{\mathrm{TM}}$, que reporta sensibilidad de $99.9 \%$ IC 95\% [94.9, 100.0\%] y especificidad de $99.9 \%$ IC $95 \%$ [95.1, 100.0\%].

Se ingresó, validó y analizó los datos en Epi Info $^{\mathrm{TM}}$ 3.5.4. Para las variables cualitativas se usó frecuencias simples y porcentajes y para las variables cuantitativas media y desviación estándar. Para el análisis de posible asociación o diferencias, para las variables cualitativas se aplicó la prueba exacta de Fisher y para las cuantitativas $t$ de Student, se consideró como significativo un valor $p<.05$.

Se solicitó consentimiento informado, el cual fue firmado por el paciente. Se contó con la aprobación del Comité de Ética del Hospital. A los pacientes que no podían costear los gastos del tratamiento se les proporcionó en forma gratuita por parte de los investigadores.

\section{Resultados}

La edad promedio (desviación estándar) de los participantes fue de 49.0 (14.4) años (rango18-79); $81.5 \%$ mujeres y $85.7 \%$ de área urbana. El síntoma más común fue dispepsia en $86.6 \%$, seguido de dolor en epigastrio en $84.9 \%$ y pirosis en $74.8 \%$ (Tabla 1 ).

En el examen post tratamiento, el 89.9\% (n = 107), IC 95\% [83.0, 94.7] presentó antígeno para $H$. pylori en heces negativo.

No se encontró diferencia significativa con la edad ni asociaciones significativas de las características evaluadas con respecto a la erradicación de $H$. pylori (Tabla 2).

Figura 1. Proceso de selección 


\section{Discusión}

La tasa de erradicación de H. pylori, con terapia triple estándar con lansoprazol, claritromicina y amoxicilina, administrada durante 10 días, en este estudio fue de $89.9 \%$, estos datos apoyan la utilización de terapia triple con claritromicina en pacientes sin tratamiento previo, como primera línea de tratamiento de erradicación.

La efectividad de la terapia triple ha experimentado una disminución en los últimos años y en los estudios más recientes ha arrojado cifras menores del $85 \%$ (Gong et al., 2014; Hu, Zhang, Lu, \& Dai, 2016; Megraud et al., 2013; Tongtawee et al., 2016). El aumento de la resistencia a los antibióticos es el determinante más importante en la falla terapéutica.

La resistencia a la terapia varía de región en región y de país a país, se reporta resistencia a la claritromicina desde $21.4 \%$ cuando se usa por primera vez, hasta $88.3 \%$ cuando se usa por segunda ocasión (Almeida et al., 2014). En Corea (Kim et al., 2014) se reporta una tasa de erradicación con terapia de primera línea (inhibidor de bomba, claritromicina y amoxicilina) del $79.9 \%$ y para segunda línea (inhibidor de bomba, bismuto, metronidazol y tetraciclina) del $90.4 \%$. Estudios en poblaciones comparables a nuestro medio han mostrado resultados con altas tasas de resistencia a la claritromicina, en Tailandia en 2014 (Tongtawee et al., 2016), la prevalencia de cepas resistentes a la claritromicina fue de $76.2 \%$ y el porcentaje de erradicación solamente alcanzó un $70 \%$, por lo que no recomiendan su uso. En Latinoamérica, la resistencia global antimicrobiana en adultos varía ampliamente para cada antibiótico y para cada país (Camargo et al., 2014; Ierardi, Giorgio, Losurdo, Di Leo, \& Principi, 2013)

Otros, sin embargo, apoyan los hallazgos reportados en el presente estudio. Un estudio aleatorio realizado en Turquía comparó tres esquemas distintos, la tasa de erradicación con claritromicina fue de $95.8 \%$ y no encontró diferencias estadísticamente significativas al comparar diferentes esquemas (Kefeli et al., 2015).

Ante el incremento de la resistencia a los tratamientos de primera línea se han realizado estudios que incluyen aumentar el número de días (Greenberg et al., 2011; Yuan et al., 2013), terapia secuencial (Chan et al., 2015; Lahbabi et al., 2013; Liou et al., 2016; Zullo, Hassan, Ridola, De Francesco, \& Vaira, 2013), concomitante (Lee et al., 2015; Leodolter et al., 2001) o terapia cuádruple (Fallone et al., 2016; Venerito, Krieger, Ecker, Leandro, \& Malfertheiner, 2013), con resultados variados. En Guatemala se estudió la erradicación con pantoprazole, levofloxacina y amoxicilina (Barahona-Garrido et al., 2012), la efectividad fue de $85 \%$ en el análisis de intención por tratamiento y $93 \%$ en el análisis por protocolo. No se encontró estudios en que se evaluara lansoprazol, amoxicilina y claritromicina en Guatemala.

El consenso de Maastricht IV (Malfertheiner et al., 2012) recomienda que en regiones donde la tasa de resistencia a la claritromicina es mayor del 15 al 20\%, se debe abandonar la terapia triple que incluya claritromicina si no se cuenta con pruebas de susceptibilidad.

Independiente del mejor esquema para la erradicación, en lo que están todos de acuerdo es en tratar la infección, sobre todo porque hay estudios que postulan que su erradicación disminuye el riesgo de cáncer (Fuccio et al., 2009), inclusive su curación está asociada a la erradicación del tumor tipo MALT (Morgner, Bayerdörffer, Neubauer, \& Stolte, 2000; Nakamura \& Matsumoto, 2013). Se recomienda incluso diagnosticar tratar a pacientes con dispepsia (Gisbert \& Calvet, 2013; Malfertheiner et al., 2012).

Tabla 1

Características generales de los participantes

\begin{tabular}{lcc}
\hline Características & $\mathrm{n}$ & $\%$ \\
\hline $\begin{array}{l}\text { Edad promedio ( DE) } \\
\text { Sexo }\end{array}$ & \multicolumn{2}{c}{$49.0(14.4)$} \\
$\quad$ Femenino & 97 & 81.5 \\
$\quad$ Masculino & 22 & 18.5 \\
Procedencia & & \\
$\quad$ Urbana & 102 & 85.7 \\
$\quad$ Rural & 17 & 14.3 \\
Síntomas & & \\
$\quad$ Dispepsia & 103 & 86.6 \\
Dolor en epigastrio & 101 & 84.9 \\
Pirosis & 89 & 74.8 \\
Náusea & 73 & 61.3 \\
Distensión & 72 & 60.5 \\
$\quad$ Saciedad temprana & 46 & 38.7 \\
$\quad$ Pérdida de peso & 17 & 14.3 \\
Hemorragia & 16 & 13.4 \\
Vómitos & 14 & 11.8 \\
\hline
\end{tabular}


Tabla 2

Erradicación de H. pylori y características generales

\begin{tabular}{|c|c|c|c|c|c|}
\hline & \multicolumn{5}{|c|}{ Erradicación de $H$. pylori } \\
\hline & \multicolumn{2}{|c|}{$\mathrm{Si}$} & \multicolumn{2}{|c|}{ No } & \\
\hline & $\mathrm{n}$ & $\%$ & $\mathrm{n}$ & $\%$ & \\
\hline Características & 107 & 89.9 & 12 & 10.1 & Valor $p$ \\
\hline Edad promedio (DE) & \multicolumn{2}{|c|}{$49.0(13.8)$} & \multicolumn{2}{|c|}{$47.0(19.6)$} & .765 \\
\hline \multicolumn{6}{|l|}{ Sexo } \\
\hline Femenino & 86 & 88.7 & 11 & 11.3 & .305 \\
\hline Masculino & 21 & 95.5 & 1 & 4.5 & \\
\hline \multicolumn{6}{|l|}{ Procedencia } \\
\hline Urbano & 92 & 90.2 & 10 & 9.8 & .538 \\
\hline Rural & 15 & 88.2 & 2 & 11.8 & \\
\hline \multicolumn{6}{|l|}{ Síntomas } \\
\hline Dispepsia & 91 & 88.3 & 12 & 11.7 & .161 \\
\hline Dolor en epigastrio & 89 & 88.1 & 12 & 11.9 & .125 \\
\hline Pirosis & 78 & 87.6 & 11 & 12.4 & .140 \\
\hline Náusea & 63 & 86.3 & 10 & 13.7 & .086 \\
\hline Distensión & 67 & 93.1 & 5 & 6.9 & .136 \\
\hline Saciedad & 40 & 87.0 & 6 & 13.0 & .291 \\
\hline Pérdida de peso & 17 & 100 & 0 & 0 & .142 \\
\hline Hemorragia & 15 & 93.8 & 1 & 6.3 & .497 \\
\hline Vómitos & 13 & 92.9 & 1 & 7.1 & .573 \\
\hline
\end{tabular}

Para la evaluación de la respuesta terapéutica existen diversos métodos, entre los no invasivos la prueba de urea en aliento ha demostrado mayor sensibilidad y especificidad (Garza-González et al., 2014), sin embargo tiene el inconveniente de su alto costo, por lo que la prueba de antígeno de H. pylori en heces es recomendado por el Consenso de Maastricht IV (Malfertheiner et al., 2012) y el Consenso Latinoamericano (Rollan et al., 2014) como una de las alternativas para evaluar la respuesta al tratamiento de erradicación de H. pylori. La sensibilidad y especificidad de la prueba de antígeno en heces depende de si se usa pre o post tratamiento, de la prevalencia del $H$. pylori y la técnica utilizada y de si monoclonal o policlonal, el monoclonal es superior (Garza-González et al., 2014; Lopes, Vale, \& Oleastro, 2014; Shimoyama, 2013), la cual fue utilizada en este estudio.
La adherencia al tratamiento es uno de los factores que puede influir en la erradicación del $H$. pylori, por lo que en este estudio se procuró asegurar la misma, sin embargo no se puede asegurar completamente, aunque debido a que el tratamiento es relativamente corto se espera una mejor adherencia. Estudios reportan tasas de adherencia de $60 \%$ al $100 \%$ en tratamiento para $H$. pylori (Fischbach et al., 2004; Lefebvre, Chang, Morse, van Zanten, \& Goodman, 2013), contrario a tratamientos prolongados donde la falta de adherencia es más alta (Briesacher, Andrade, Fouayzi, \& Chan, 2008; Claxton, Cramer, \& Pierce, 2001; Hugtenburg, Timmers, Elders, Vervloet, \& van Dijk, 2013).

En este estudio sólo un paciente manifestó efectos adversos pero se excluyó debido a que suspendió el tratamiento, por lo que no se pudo relacionar los efectos adversos con la erradicación del $H$. pylori. 
Aunque la tasa de erradicación alcanzada se encuentra entre los valores aceptados para continuar con el esquema utilizado, se debe estudiar los factores que pueden estar asociados a la respuesta, para ello se tendrá que proponer otro tipo de diseño que permita identificar esos factores, además de la resistencia a los antibióticos en los que no responden al tratamiento.

En conclusión, la tasa de erradicación de $H$. pylori en pacientes que recibieron terapia triple estándar con lansoprazol, claritromicina y amoxicilina, fue de $89.9 \%$ y no se encontró asociaciones significativas entre las características demográficas o síntomas asociados con respecto a la tasa erradicación, por lo que se recomienda la terapia triple estándar (claritromicina, lansoprazol, amoxicilina) como terapia de primera línea de erradicación en pacientes sin tratamiento previo, mientras no se demuestre un incremento de la resistencia a dicho esquema, aunque lo recomendable es mantener una evaluación permanente del comportamiento de la bacteria a la terapia triple.

Conflicto de intereses: Los autores declaran no tener ningún conflicto de intereses.

\section{Referencias}

Almeida, N., Romãozinho, J. M., Donato, M. M., Luxo, C., Cardoso, O., Cipriano, M. A., ... Sofia, C. (2014). Helicobacter pylori antimicrobial resistance rates in the central region of Portugal. Clinical Microbiology and Infection, 20(11), 11271133. doi: 10.1111/1469-0691.12701

Barahona-Garrido, J., Ortiz, J. C., \& Sarti, H. M. (2012). Pantoprazol, levofloxacina y amoxicilina como tratamiento de primera línea para la erradicación de Helicobacter pylori en Guatemala: efectividad en un país en vías de desarrollo. Endoscopia, 24(4), 154-159.

Barahona-Garrido, J., Quiñonez, N. F., Cerda-Contreras, E., Maria Sarti, H., \& Téllez-Ávila, F. I. (2013). Fosfomycin-containing second-line treatment for Helicobacter pylori infection. American Journal of Gastroenterology, 108(5), 858-9. doi: 10.1038/ajg.2013.48

Benajah, D. A., Lahbabi, M., Alaoui, S., El Rhazi, K., El Abkari, M., Nejjari, C., ... Ibrahimi, S. A. (2013). Prevalence of Helicobacter pylori and its recurrence after successful eradication in a developing nation (Morocco). Clinics and Research in Hepatology and Gastroenterology, 37(5), 519526. doi: 10.1016/j.clinre.2013.02.003

Briesacher, B. a, Andrade, S. E., Fouayzi, H., \& Chan, K. A. (2008). Comparison of Drug Adherence Rates Among Patients with Seven Different Medical Conditions. Pharmacotherapy, 28(4), 432-443. doi: 10.1592/phco.28.4.437

Camargo, M. C., Garcia, A., Riquelme, A., Otero, W., Camargo, C. A., Hernandez-Garcia, T., ... Rabkin, C. S. (2014). The problem of Helicobacter pylori resistance to antibiotics: a systematic review in Latin America. American Journal of Gastroenterology, 109(4), 485-495. doi: 10.1038/ ajg.2014.24

Chan, C.-C., Chien, N.-H., Lee, C.-L., Yang, Y.-C., Hung, C.-S., Tu, T.-C., \& Wu, C.-H. (2015). Comparison of 10-day sequential therapy with 7-day standard triple therapy for Helicobacter pylori eradication in inactive peptic ulcer disease and the efficiency of sequential therapy in inactive peptic ulcer disease and non-ulcer dyspepsia. BMC Gastroenterology, 15(1), 170. doi: 10.1186/ s12876-015-0401-4

Chey, W. D., Leontiadis, G. I., Howden, C. W., \& Moss, S. F. (2017). ACG Clinical Guideline: Treatment of Helicobacter pylori Infection. The American Journal of Gastroenterology, 112(2), 212-239. doi: 10.1038/ajg.2016.563

Chey, W. D., Wong, B. C. Y., \& Practice Parameters Committee of the American College of Gastroenterology. (2007). American College of Gastroenterology Guideline on the Management of Helicobacter pylori Infection. The American Journal of Gastroenterology, 102(8), 1808-1825. doi: 10.1111/j.1572-0241.2007.01393.x

Claxton, A. J., Cramer, J., \& Pierce, C. (2001). A systematic review of the associations between dose regimens and medication compliance. Clinical Therapeutics, 23(8), 1296-1310. doi: 10.1016/ S0149-2918(01)80109-0

Eusebi, L. H., Zagari, R. M., \& Bazzoli, F. (2014). Epidemiology of Helicobacter pylori infection. Helicobacter, 19(S1), 1-5. doi: 10.1111/hel.12165 
Fallone, C. A., Chiba, N., van Zanten, S. V., Fischbach, L., Gisbert, J. P., Hunt, R. H., ... Marshall, J. K. (2016). The Toronto Consensus for the Treatment of Helicobacter pylori Infection in Adults. Gastroenterology, 151(1), 51-69.e14. doi: 10.1053/j. gastro.2016.04.006

Fischbach, L. A., Van Zanten, S. V., \& Dickason, J. (2004). Meta-analysis: The efficacy, adverse events, and adherence related to first-line anti-Helicobacter pylori quadruple therapies. Alimentary Pharmacology and Therapeutics, 20(10), 10711082. doi: 10.1111/j.1365-2036.2004.02248.x

Fock, K. M., Katelaris, P., Sugano, K., Ang, T. L., Hunt, R., Talley, N. J., ... Rani, A. A. (2009). Second Asia-Pacific Consensus Guidelines for Helicobacter pylori infection. Journal of Gastroenterology and Hepatology (Australia), 24(10), 15871600. doi: 10.1111/j.1440-1746.2009.05982.x

Fuccio, L., Zagari, R. M., Eusebi, L. H., Laterza, L., Cennamo, V., \& Ceroni, L. (2009). Meta-analysis: Can Helicobacter pylori eradication treatment reduce the risk for gastric cancer?. Annals of Internal Medicine, 151(7), 121-128.

Garza-González, E., Perez-Perez, G. I., Maldonado-Garza, H. J., \& Bosques-Padilla, F. J. (2014). A review of Helicobacter pylori diagnosis, treatment, and methods to detect eradication. World Journal of Gastroenterology, 20(6), 1438-1449. doi: 10.3748/wjg.v20.i6.1438

Garza González, E., Giasi González, E., Martínez Vázquez, M. a, Pérez Pérez, G. I., González, G. M., Maldonado Garza, H. J., \& Bosques Padilla, F. J. (2007). Helicobacter pylori erradication and its relation to antibiotic resistance and CYP2C19 status. Revista Española de Enfermedades Digestivas : Organo Oficial de La Sociedad Espanola de Patologia Digestiva, 99(2), 71-75.

Gisbert, J. P., \& Calvet, X. (2013). Helicobacter Pylo$r i$ "Test-and-Treat" Strategy for Management of Dyspepsia: A Comprehensive Review. Clinical and Translational Gastroenterology, 4(3), e32. doi: 10.1038/ctg.2013.3

Gong, E. J., Yun, S.-C., Jung, H.-Y., Lim, H., Choi, K.S., Ahn, J. Y., ... Kim, J.-H. (2014). Meta-Analysis of First-Line Triple Therapy for Helicobacter pylori Eradication in Korea: Is It Time to Change?
Journal of Korean Medical Science, 29(5), 704713. doi: 10.3346/jkms.2014.29.5.704

Greenberg, E. R., Anderson, G. L., Morgan, D. R., Torres, J., Chey, W. D., Bravo, L. E., ... Baker, L. H. (2011). 14-day triple, 5-day concomitant, and 10-day sequential therapies for Helicobacter pylori infection in seven Latin American sites: a randomised trial. Lancet, 378(9790), 507-514. doi: 10.1016/S0140-6736(11)60825-8

Hu, Y., Zhang, M., Lu, B., \& Dai, J. (2016). Helicobacter pylori and Antibiotic Resistance, A Continuing and Intractable Problem. Helicobacter, 21(5), 349-363. doi: 10.1111/hel.12299

Hugtenburg, J. G., Timmers, L., Elders, P. J. M., Vervloet, M., \& van Dijk, L. (2013). Definitions, variants, and causes of nonadherence with medication: A challenge for tailored interventions. Patient Preference and Adherence, 7, 675-682. doi: 10.2147/PPA.S29549

Hunt, R. H., Xiao, S. D., Megraud, F., Leon-Barua, R., Bazzoli, F., van der Merwe, S., ... le Mair, A. (2011). Helicobacter pylori in developing countries. World gastroenterology organisation global guideline. Journal of Gastrointestinal and Liver Diseases, 20(3), 299-304. doi: 10.1097/MCG. 0b013e31820fb8f6

Ierardi, E., Giorgio, F., Losurdo, G., Di Leo, A., \& Principi, M. (2013). How antibiotic resistances could change Helicobacter pylori treatment: A matter of geography? World Journal of Gastroenterology, 19(45), 8168-8180. doi: 10.3748/wjg.v19. i45.8168

Kefeli, A., Basyigit, S., Yeniova, A. O., Kefeli, T. T., Aslan, M., \& Tanas, O. (2015). Comparison of three different regimens against Helicobacter pylori as a first-line treatment: A randomized clinical trial. Bosnian Journal of Basic Medical Sciences, 13-16. doi: 10.17305/bjbms.2016.660

Kim, S. Y., Hyun, J. J., Jung, S. W., Koo, J. S., Yim, H. J., \& Lee, S. W. (2014). Helicobacter pylori Recurrence after First- and Second-Line Eradication Therapy in Korea: The Problem of Recrudescence or Reinfection. Helicobacter, 19(3), 202-206. doi: 10.1111/hel.12117

Lahbabi, M., Alaoui, S., El Rhazi, K., El Abkari, M., 
Nejjari, C., Amarti, A., ... Benajah, D. A. (2013). Sequential therapy versus standard triple-drug therapy for Helicobacter pylori eradication: Result of the HPFEZ randomised study. Clinics and Research in Hepatology and Gastroenterology, 37(4), 416-421. doi: 10.1016/j.clinre.2012.10.002

Lee, H. J., Kim, J. Il, Lee, J. S., Jun, E. J., Oh, J. H., Cheung, D. Y., ... Kim, S. S. (2015). Concomitant therapy achieved the best eradication rate for $\mathrm{He}$ licobacter pylori among various treatment strategies. World Journal of Gastroenterology, 21(1), 351-359. doi: 10.3748/wjg.v21.i1.351

Lefebvre, M., Chang, H., Morse, A., van Zanten, S. V., \& Goodman, K. J. (2013). Adherence and barriers to H. pylori treatment in Arctic Canada. International Journal of Circumpolar Health, 72(1), 22791. doi: 10.3402/ijch.v72i0.22791

Leodolter, A., Kulig, M., Brasch, H., Meyer-Sabellek, W., Willich, S. N., \& Malfertheiner, P. (2001). A meta-analysis comparing eradication, healing and relapse rates in patients with Helicobacter pylo$r i$-associated gastric or duodenal ulcer. Alimentary Pharmacology and Therapeutics, 15(12), 19491958. doi: 10.1046/j.1365-2036.2001.01109.x

Li, B.-Z., Threapleton, D. E., Wang, J.-Y., Xu, J.-M., Yuan, J.-Q., Zhang, C., ... Ye, D.-Q. (2015). Comparative effectiveness and tolerance of treatments for Helicobacter pylori : systematic review and network meta-analysis. $B M J, \mathrm{~h} 4052$. doi: 10.1136/bmj.h4052

Liou, J.-M., Chen, C.-C., Chang, C.-Y., Chen, M.-J., Chen, C., Fang, Y., ... Wu, M.-S. (2016). Sequential therapy for 10 days versus triple therapy for 14 days in the eradication of Helicobacter pylo$r i$ in the community and hospital populations: a randomised trial. Gut, 65(11), 1784-1792. doi: 10.1136/gutjnl-2015-310142

Lopes, A. I., Vale, F. F., \& Oleastro, M. (2014). Helicobacter pylori infection - Recent developments in diagnosis. World Journal of Gastroenterology, 20(28), 9299-9313. doi: 10.3748/wjg.v20. i28.9299

Malfertheiner, P., Megraud, F., O'Morain, C. A., Atherton, J., Axon, A. T. R., Bazzoli, F., ... The European Helicobacter Study Group. (2012). Management of Helicobacter pylori infection - the
Maastricht IV/ Florence Consensus Report. Gut, 61(5), 646-664. doi: 10.1136/gutjnl-2012-302084 Malfertheiner, P., Megraud, F., O’Morain, C. A., Gisbert, J. P., Kuipers, E. J., Axon, A. T., ... El-Omar, E. M. (2017). Management of Helicobacter pylori infection - the Maastricht V/Florence Consensus Report. Gut, 66(1), 6-30. doi: 10.1136/gutjnl-2016-312288

Megraud, F., Coenen, S., Versporten, A., Kist, M., Lopez-Brea, M., Hirschl, A. M., ... Glupczynski, Y. (2013). Helicobacter pylori resistance to antibiotics in Europe and its relationship to antibiotic consumption. Gut, 62(1), 34-42. doi: 10.1136/ gutjnl-2012-302254

Mentis, A., Lehours, P., \& Mégraud, F. (2015). Epidemiology and Diagnosis of Helicobacter pylori infection. Helicobacter, 20, 1-7. doi: 10.1111/ hel. 12250

Morgner, A., Bayerdörffer, E., Neubauer, A., \& Stolte, M. (2000). Malignant tumors of the stomach. Gastric mucosa-associated lymphoid tissue lymphoma and Helicobacter pylori. Gastroenterology Clinics of North America, 29(3), 593-607. doi: 10.1016/50889-8553 (05) 70132-1

Nakamura, S., \& Matsumoto, T. (2013). Helicobacter pylori and gastric mucosa-associated lymphoid tissue lymphoma: Recent progress in pathogenesis and management. World Journal of Gastroenterology, 19(45), 8181-8187. doi: 10.3748/ wjg.v19.i45.8181

O'Connor, A., Vaira, D., Gisbert, J. P., \& O’Morain, C. (2014). Treatment of Helicobacter pylori infection 2014. Helicobacter, 19(S1), 38-45. doi: 10.1111/hel.12163

Parkin, D. M. (2006). The global health burden of infection-associated cancers in the year 2002. International Journal of Cancer, 118(12), 3030-3044. doi: 10.1002/ijc. 21731

Plummer, M., Franceschi, S., Vignat, J., Forman, D., $\&$ De Martel, C. (2015). Global burden of gastric cancer attributable to Helicobacter pylori. International Journal of Cancer. 136(2), 487-490. doi: 10.1002/ijc. 28999

Prell, C., Osterrieder, S., Lottspeich, C., Schwarzer, A., Rüssmann, H., Ossiander, G., \& Koletzko, S. (2009). Improved performance of a rapid offi- 
ce-based stool test for detection of Helicobacter pylori in children before and after therapy. Journal of Clinical Microbiology, 47(12), 3980-3984. doi: 10.1128/JCM.01204-09

Rollan, A., Arab, J. P., Camargo, M. C., Candia, R., Harris, P., Ferreccio, C., ... Riquelme, A. (2014). Management of Helicobacter pylori infection in Latin America: A Delphi technique-based consensus. World Journal of Gastroenterology, 20(31), 10969-10983. doi: 10.3748/wjg.v20.i31.10969

Schneider, R. E., Vettorazzi, M., Torres, M. A., Solís, C., Marroquín, S., Morales, H., \& Rodríguez, E. (1994). La infección gástrico por Helicobacter pylori en adultos dispépticos de Guatemala. $\mathrm{Su}$ relación con el estado socio-económico y cambios displásicos gástricos. Revista Medicina Inerna, 5(1), 2-9.

Shimoyama, T. (2013). Stool antigen tests for the management of Helicobacter pylori infection. World Journal of Gastroenterology, 19(45), 8188-8191. doi: 10.3748/wjg.v19.i45.8188

Sugano, K., Tack, J., Kuipers, E. J., Graham, D. Y., El-Omar, E. M., Miura, S., ... Malfertheiner, P. (2015). Kyoto global consensus report on Helicobacter pylori gastritis. Gut, 1-15. doi: 10.1136/ gutjnl-2015-309252

Suzuki, T., Matsuo, K., Sawaki, A., Ito, H., Hirose, K., Wakai, K., ... Tajima, K. (2006). Systematic review and meta-analysis: Importance of CagA status for successful eradication of Helicobacter pylori infection. Alimentary Pharmacology and Therapeutics, 24(2), 273-280. doi: 10.1111/j.1365-2036.2006.02994.x

Thung, I., Aramin, H., Vavinskaya, V., Gupta, S., Park, J. Y., Crowe, S. E., \& Valasek, M. A. (2016). Review article: The global emergence of Helicobac- ter pylori antibiotic resistance. Alimentary Pharmacology and Therapeutics, 43(4), 514-533. doi: 10.1111/apt.13497

Tongtawee, T., Dechsukhum, C., Matrakool, L., Panpimanmas, S., Loyd, R. A., Kaewpitoon, S. J., \& Kaewpitoon, N. (2016). High prevalence of Helicobacter pylori resistance to clarithromycin: A hospital-based cross-sectional study in Nakhon Ratchasima Province, Northeast of Thailand. Asian Pacific Journal of Cancer Prevention, 16(18), 8281-8285. doi: 10.7314/APJCP.2015.16.18.8281

Venerito, M., Krieger, T., Ecker, T., Leandro, G., \& Malfertheiner, P. (2013). Meta-analysis of bismuth quadruple therapy versus clarithromycin triple therapy for empiric primary treatment of Helicobacter pylori infection. Digestion, 88(1), 33-45. doi: 10.1159/000350719

Yuan, Y., Ford, A. C., Khan, K. J., Gisbert, J. P., Forman, D., Leontiadis, G. I., ... Moayyedi, P. (2013). Optimum duration of regimens for Helicobacter pylori eradication. Cochrane Database of Systematic Reviews, 12(12), CD008337. doi: 10.1002/14651858.CD008337.pub2

Zullo, A., Hassan, C., Ridola, L., De Francesco, V., \& Vaira, D. (2013). Standard triple and sequential therapies for Helicobacter pylori eradication: An update. European Journal of Internal Medicine, 24(1), 16-19. doi: 10.1016/j.ejim.2012.07.006 